\title{
Germanica
}

\section{Crises des rapports interpersonnels dans le roman Auto-da-fé d'Élias Canetti. Apocalypse et phobie du bleu}

Krisen in den zwischenmenschlichen Beziehungen in Elias Canettis Roman Die

Blendung. Apokalyptisches Nachplappern und ozeanische Angst vor der

Muschel.

Éric Leroy du Cardonnoy

\section{(2) OpenEdition}

\section{Journals}

Édition électronique

URL : http://journals.openedition.org/germanica/1321

DOI : 10.4000/germanica. 1321

ISSN : 2107-0784

\section{Éditeur}

Université de Lille

\section{Édition imprimée}

Date de publication : 30 juin 1998

Pagination : 79-91

ISBN : $9770984263203-22$

ISSN : 0984-2632

\section{Référence électronique}

Éric Leroy du Cardonnoy, «Crises des rapports interpersonnels dans le roman Auto-da-fé d'Élias

Canetti. Apocalypse et phobie du bleu », Germanica [En ligne], 22 | 1998, mis en ligne le 31 janvier 2012, consulté le 06 octobre 2020. URL : http://journals.openedition.org/germanica/1321 ; DOI : https:// doi.org/10.4000/germanica. 1321

Ce document a été généré automatiquement le 6 octobre 2020.

(C) Tous droits réservés 


\title{
Crises des rapports interpersonnels dans le roman Auto-da-fé d'Élias Canetti. Apocalypse et phobie du bleu
}

\author{
Krisen in den zwischenmenschlichen Beziehungen in Elias Canettis Roman Die \\ Blendung. Apokalyptisches Nachplappern und ozeanische Angst vor der \\ Muschel.
}

Éric Leroy du Cardonnoy

1 Toute la production littéraire d'Elias Canetti est placée sous le signe du conflit inéluctable et $\mathrm{du}$ combat sans merci; son histoire personnelle, telle que l'autobiographie nous la présente, n'échappe pas à cette règle. Que les hommes aient entre eux des liens de parenté ou non, cette considération n'entre pas en ligne de compte : le dessein de chacun d'entre nous, être réel comme personnage fictif, est de s'imposer au détriment d'autrui, mais aussi et surtout, de manière paradoxale, de soimême. Pour cela différentes tactiques et stratégies sont mises en place par le sujet consciemment et inconsciemment, même si, aux yeux d'Elias Canetti, grand pourfendeur des moulins psychanalytiques, ce second terme ne veut rien dire.

2 Sa première œuvre publiée date de 1932, il s'agit de Noce ${ }^{1}$; le " personnage » principal en est la maison dans laquelle l'action a lieu et que tout le monde convoite, mais qui finalement s'écroulera. Le perroquet de la propriétaire La Garpe, crie dans la première et dernière scène : «Maison! maison! maison!", sans en comprendre évidemment la portée. Mais il n'est pas le seul dans ce cas, Michel ou Bileux, par exemple, se trouvent à plusieurs reprises dans la même situation. Voilà donc pour la première partie de notre titre. Le deuxième membre fait, lui, référence à l'unique roman d'Elias Canetti, Auto-da$f^{2}$, qui date de 1935 et met en scène le personnage de Peter Kien, sinologue de profession, mais sans charge d'enseignement; ce serait en effet, dans les représentations de sa mégalomanie pathologique, déroger que de perdre du temps à 
s'adresser à des êtres incapables de s'élever pour comprendre les raffinements des textes anciens, c'est-à-dire tout simplement les autres hommes, mais aussi les femmes. Kien est en effet aux prises avec sa gouvernante, Thérèse Krumbholz, dont la laideur rivalise avec sa stupidité bornée et dont la jupe bleue amidonnée devient au cours de l'évolution catastrophique de leurs relations un objet fétiche qui permet à Kien de conjurer sa peur de Thérèse. Il ira même jusqu'à dénier à celle-ci toute existence indépendante en la réduisant à une moule qu'il a, étant jeune, écrasée sur une plage dans un accès de rage parce qu'il ne parvenait pas à l'ouvrir (chapitre intitulé «la moule " p. 57-69): la jupe bleue amidonnée devient Thérèse et cache la moule effrayante ("Thérèse sans sa coquille, sans sa jupe, n'existait pas » p. 64). Kien, pour faire un jeu de mots, a une "peur bleue » de la moule ! La victoire sur la moule indique déjà la pente que prendra le délire kienesque et les antagonismes entre les hommes, les sexes et la violence qui leur est sous-jacente.

Ces deux œuvres correspondent de plus à la période qui précède l'exil auquel l'auteur fut contraint après l'Anschluss en 1938 et peuvent être considérées comme les traces du climat politique et culturel de l'époque, mais sont aussi le témoignage de l'influence d'une certaine vision du monde sur le jeune auteur Elias Canetti et de certaines expériences déterminantes pour sa vie ${ }^{3}$. Trois axes guideront ici notre exposé, parce qu'ils forment en quelque sorte les jalons que reprendra en les développant l'œuvre ultérieur de l'auteur. Nous nous intéresserons dans un premier temps aux rapports que les hommes entretiennent avec la langue. Nous indiquerons ensuite comment la négation du corps, ou son contraire, mène à un enfermement réel ou métaphorique, en affirmant que ce qui clôture les deux œuvres, c'est-à-dire d'une part une fin du monde, d'autre part un incendie gigantesque, peut être une solution, à moins que ce ne soit la trace d'un désespoir et d'un scepticisme généralisés. Il s'agit en effet de la destruction et de la disparition du sujet tel que les Lumières, dans leur désir d'autonomie, d'indépendance et de scientificité, l'avaient élaboré, autrement dit, il est bien question d'un aveu d'échec.

\section{Les hommes et la langue}

4 La polémologie des sexes qui se manifestent dans toute sa crudité à travers la pièce comme à travers le roman est la mise en pratique et l'illustration des théories weiningériennes présentées dans Sexe et caractère de 1903. Kien se vante d'ailleurs d'être un «vrai caractère » et de pouvoir ainsi mener sa vie en pure intellectualité. Certains critiques comme Kristie A. Foell, Elfriede Pöder et Gerald Stieg ${ }^{4}$ ont démontré qu'Auto-da-fé était à lire comme une illustration satirique des thèses d'Otto Weininger, notamment en ce qui concerne la génialité - ou caractère - et la misogynie fondamentale de sa théorie. En effet, celle-ci affirme que le monde est gouverné par deux principes antagonistes, le principe masculin et le principe féminin, et que leur destin consiste dans l'éradication du second par le premier. La folie de Kien deviendra telle qu'il finira par croire envers et contre tout avoir tué sa femme, Thérèse.

5 Cette polémologie se retrouve dans la langue : Kien affirme dans un élan de maîtrise, avant que Thérèse ne le chasse de son paradis livresque, que «la langue en fin de compte est là pour l'homme et non pas l'homme pour la langue » (p. 195). À la suite de quoi, il décrète qu'il n'y a plus désormais «die Vergangenheit», mais «der Vergangenheit » et «[ $\left[c^{\prime}\right]$ est pourquoi il priait le Passé, au masculin, de bien vouloir 
approuver cette modification » (p. 195). De la sorte, Kien abolit l'existence du féminin comme genre grammatical et par là-même de Thérèse comme représentation et incarnation du genre animé. D'ailleurs le délire de Kien s'attachera à une espèce animale qui force son admiration par qu'elle a réussi à surmonter le clivage des sexes : les termites, dont on imagine le danger qu'elles représentent pour le propriétaire d'une bibliothèque de 25000 volumes! Cette abolition de la différenciation sexuelle signifie dans le même temps la disparition pure et simple de ces insectes.

6 L'homme dominerait donc la langue et par conséquent le monde, mais nous allons voir que cette domination n'est en fait qu'un leurre dont chaque homme s'empare avec délectation afin de conjurer la peur de la vie. Les hommes ne dominent pas la langue, ils sont parlés par elle ${ }^{5}$ ils sont un objet insignifiant dans les rouages de l'appareil linguistique. Ainsi les personnages parlent de manière générale, c'est-à-dire que chaque personnage de la pièce et du roman ne possède pas les mots qu'il emploie, mais se trouve à leur merci, contrairement à ce qu'il croit ou affirme lui-même. Ainsi p. 78 et p. 130 par exemple Kien ne peut pas dire « je », il dit « on », c'est-à-dire qu'il succombe à la disparition de son identité, dans les livres. Il déclare lui-même qu'il « préfère plutôt s'exprimer par écrit que par oral, en paragraphes longuement médités » (p. 19), c'est-àdire en fait par des citations tirées de ses lectures. Tous les personnages pensent par formules, la langue semble refuser de se transformer en parole. L'utilisation des sociolectes (sentences populaires chez Thérèse, argot criminel dans le monde de Fischerle, jargon policier de Pfaff, dialecte viennois de la vieille La Garpe, jargon pseudo-académique de Parfait, etc.) marque également à quel point chacun est victime de son milieu et soumis à une vision du monde dont il n'a pas pleine conscience et sur laquelle il n'a finalement aucun contrôle.

7 Si Kien interdit à Thérèse de parler pendant les repas par contrat synallagmatique, c'est qu'il ne supporte pas ses discours creux, faits de formules toutes faites et répétitives, sensés exprimer le sens commun, c'est-à-dire dans le cas de Thérèse, une vision du monde petite-bourgeoise étriquée qu'elle veut imposer au monde pour le comprendre et donc le maîtriser. Le silence que lui impose son employeur, devenu mari, ne vise qu'à la maintenir à distance - après l'échec copulatif de monsieur et madame devant l'état civil, et copulatoire devant l'état physique des deux. Or, Thérèse aime parler ("L'obligation de garder le silence lui était plus dure que le silence lui-même.» p. 71), en fait se répéter constamment; l'interdit ne fait par conséquent que provoquer une accumulation explosive de ce qui est réprimé, ce trop-plein se déchargeant dans l'épisode qui clôt la première partie du roman où Thérèse empoigne Kien, le bat comme plâtre et le chasse de l'appartement avant de sombrer dans une orgie de monologues non interruptus.

8 Comme nous le disions plus haut, chacun voudrait maîtriser le monde et les autres hommes, et la langue est dans cette entreprise à la fois ancillaire et maitresse. Ainsi les personnages rebaptisent les autres: Thérèse transforme "l'homme intéressant ", le vendeur de meubles Grob, en Monsieur Puda, qu'elle dérive de Buddah, un nom qu'elle est parvenue à déchiffrer sur la reliure d'un volume de la bibliothèque au cours de son ménage quotidien. En fait, dans ce cas, nous trouvons peut-être la plus belle illustration $\mathrm{du}$ fait que la langue gouverne les hommes ce dont ils ne sont pas, ou ne veulent pas être, conscients. En effet le nom qu'elle donne à son amant fantasmé - celui-ci n'a employé avec elle le ton obséquieux et les compliments faciles qu'afin de lui vendre l'ameublement de la chambre nuptiale (p.117-122) - est tiré du verbe dialectal 
«pudern" qui signifie «faire l'amour » et donne à entendre les qualités illusoires que Thérèse confère à cet homme et le programme qu'elle se donne. Le nom est le reflet de son désir de vieille fille frustrée. Elle n'est pas la seule dans ce cas, tous les personnages en font autant. Dans Noce, par exemple, c'est dès le premier tableau Tonine qui alors que sa grand-mère lui demande si elle s'est laissée embrasser par l'homme dans l'escalier, lui répond abruptement que le chat joue encore avec la laine. A cet endroit, de nouveau la langue détermine l'homme, puisqu'ici la référence au chat est due à l'homophonie de «Bussi » (prononcé en viennois «Pussi ») et de « die Pussi » pour le chat. Dans les deux cas, il y a neutralisation de l'opposition entre les consonnes sourdes et sonores au profit de la sonore, un glissement par déviation et dérivation individuelle certes, mais inscrite dans la langue. Même s'il semble s'agir à l'origine d'un désir de prise de possession de l'autre ou plus exactement d'une négation de l'autre par intégration totale à la propre logique de son désir en lui niant une complexité propre, il s'agit bien plus d'une manipulation par la langue elle-même ce qui vient démentir totalement l'affirmation citée plus haut de Kien. Dans les deux cas présentés, nous avons donc affaire à un «durcissement » consonantique, homologue de la dureté des rapports entre les hommes.

D'autre part la langue possède une qualité incomparable pour tous les personnages, parce qu'elle semble créer les moindres désirs de chacun: c'est sa possibilité performative, étendue dans notre cas à toute la langue et non plus réservée seulement à certains verbes. Ainsi Kien se crée une bibliothèque «dans la tête » qu'il transporte partout avec lui et la force de conviction de Fischerle ne fait que renforcer cette hallucination. Le portier de l'hôtel, où Kien passe la nuit après avoir été chassé par Thérèse, fait de lui le propriétaire de la bibliothèque de la cour. Fischerle invente la mort de Thérèse à laquelle croit Kien et dont ce dernier se croit responsable: il aurait enfermé Thérèse dans la bibliothèque où elle se serait auto-dévorée. Fischerle finit d'ailleurs lui-même par croire à ce récit d'une mort inventée (p. 320). Cette mort est racontée deux fois, à quelques chapitres d'intervalle, mais entre temps le délire de Kien est devenu tel que plus rien ne peut l'arrêter. Ainsi la seconde mort de Thérèse devient cosmique puisque sa mort a été suivie de la disparition totale de son cadavre :

Elle s'est dévorée elle-même. Son corps, morceau par morceau, a été la proie de son avidité. Elle maigrissait de jour en jour. Elle était trop faible pour se lever et restait couchée dans ses propres déjections. [...] Un jour, elle expira. Même cette expression dénature la vérité ; elle n'avait sans doute plus de poumons, [...] Elle était couverte d'ordures. Sa chair déchirée, qu'elle arrachait en lambeaux de tout son corps, empestait jusqu'au ciel. La putréfaction commença alors qu'elle vivait encore [...]. Une clameur sauvage gagnait de rue en rue. Des hommes indignés quittèrent leur travail, des femmes étaient prises de crises de larmes, les écoles crachaient leurs enfants, par milliers les gens s'assemblaient et exigeaient qu'on fasse tuer le cadavre. Depuis la Révolution de 48 , on n'avait pas vu pareille émeute. Des poings dressés, des jurons, des rues haletantes, des voix qui crient en cœur : «Mort au cadavre! Mort au cadavre! » Je comprends cela. La foule est instable. En général, je ne l'aime pas. Pourtant, avec quelle joie je me serais mêlé à elle ce jourlà ! Le peuple n'entend pas la plaisanterie. Terrible est sa vengeance [...], p. 382-386.

Pour Kien, donc, raconter la mort de sa femme équivaut à la mort réelle de celle-ci, la narration de son décès le rend réel. C'est pourquoi lors de l'interrogatoire au poste de police il refuse de regarder derrière son dos où elle se trouve, puisqu'ainsi, ne la voyant pas, elle n'existe plus pour lui. Kien poussera la folie jusqu'à vérifier l'exactitude de son hallucination, en l'occurrence la présence physique réelle de Thérèse, par l'existence de 
Thérèse qui se tient dans la même pièce, exactitude qui prouvera, selon lui, que Thérèse est bien morte ; autrement dit la réalité confirme l'hallucination au lieu de l'infirmer. L'utilisation du conditionnel est aussi un élément important, car il rend l'hypothétique possible, il permet aux personnages de vivre dans une réalité que l'on pourrait nommer une réalité virtuelle. C'est le cas par exemple de Kien p.19, lorsqu'il explique son attitude vis-à-vis des colloques où il refuse de se rendre : il vit dans un monde parallèle de mégalomane. Encore cette affirmation demanderait-elle à être nuancée. En effet l'utilisation du style indirect libre par Elias Canetti brouille les pistes, non seulement pour le lecteur, mais aussi pour le personnage lui-même qui ne sait plus quand il est entré dans cette réalité virtuelle et qui, s'y sentant à son aise, puisqu'elle obéit à ses désirs, décide de ne plus la quitter.

11 La langue qui est supposée permettre le contact entre les hommes subit un remarquable échec. Ici elle ne sert qu'à exprimer le désir profond de chacun au détriment d'une écoute de l'autre. À cet égard les « paupières » que Kien se laisse soitdisant pousser sur les oreilles (p.131) sont révélatrices de cet état de fait: elles lui permettent de s'isoler à volonté du monde, de ne pas entendre ce que lui dit sa femme. Les dialogues sont presque toujours des dialogues simulés, tel celui que Kien engage avec Confucius au moment où il hésite encore à se marier (p. 53-56). Il n'y a dans Autoda-fé, mais aussi dans Noce, que des monologues qui équivalent à des dialogues de sourds, nous citerons par exemple la tentative désespérée de la vieille Kokosch d'avoir une dernière conversation avant de mourir avec son mari qui lui récite des passages de la Bible à haute voix sans prêter attention à la dernière volonté de sa femme (p. 24-26 et p. 73) ou bien encore la discussion entre Kien et Thérèse sur le million à hériter où chacun s'approprie l'héritage que l'autre suppose faire de la mort du premier. Lorsqu'enfin ils s'aperçoivent de la méprise, c'est de part et d'autre l'épuisement physique - qui les empêche d'en venir aux mains - et la déception, et le narrateur de conclure : "Quelques instants plus tard ils s'étaient compris pour la première fois. " (p. 176)!

12 Les monologues, que chaque personnage produit, sont en fait empreints d'une circularité qui traduit l'enfermement où ils se trouvent, que ce soit l'enfermement de Kien dans sa bibliothèque véritable ou sa "bibliothèque de tête » ou le désir charnel de Bouc pour toutes les femmes qu'il rencontre. Tous sont pris dans les méandres de leur désir, de leur "mythe privé » qui les empêche de s'ouvrir aux autres. L'ouverture lorsqu'elle a lieu, par exemple lorsque Kien ouvre sa bibliothèque à Thérèse, n'a pour conséquence qu'un désastre, car elle se produit sur un malentendu flagrant : il pense qu'elle attache de la valeur aux livres alors qu'elle n'attache d'importance qu'à la valeur marchande de ses livres. En effet, celui-ci comprend tout a priori dans un sens figuré, alors qu'elle évolue dans un monde d'où la connotation et la métaphore sont bannies, elle prend tout au pied de la lettre. Leurs relations sont placées, de ce fait, sous le sceau de l'incompréhension originelle.

13 Il en va de même pour le personnage de Georges, le frère de Kien qui dirige un asile à Paris. Lorsqu'il arrive à Vienne pour aider son frère, il décide de le faire parler, mais il s'agit en fait de ne pas mettre en péril sa propre situation et sa propre renommée - que penserait-on d'un directeur d'asile dont le frère serait fou ? - plutôt que d'aider son frère. Et Canetti en profite pour porter un rude coup à la psychanalyse, car ce qu'effectue Georges est de fait une écoute flottante où il n'entend que ce qui peut le conforter dans son présupposé quant à l'état de son frère (chapitre intitulé «Le rusé 
Ulysse » p. 515 et suivantes). Ou bien c'est encore l'explication du succès de Georges comme directeur d'asile: il ne cherche en effet pas à guérir ses patients, mais les entretient dans leur folie en faisant en sorte qu'ils s'y sentent bien; il peut ainsi régner sur son asile sans contestation. La folie n'est qu'une manière inadaptée de vivre dans le monde, une impossibilité fondamentale à communiquer son malheur et sa douleur (p. 488). Georges préfère y voir - non sans cynisme - un monde parallèle dans lequel ses malades sont finalement heureux. Il va même jusqu'à penser que "[...] sans la possession de ces proches que l'on torture et que l'on aime, nul ne pense que la vie vaille la peine d'être vécue. » (p. 488)

\section{Le refus ou l'exaltation du corps et de ses désirs}

Ici les deux œuvres choisies comme objet de notre étude forment les deux pans d'une position face au corps et au désir. Dans Noce, tous les personnages sont mus par leur désir charnel pour l'autre et l'étalé au grand jour, même si celui-ci devrait être exclu du champ de ce même désir du fait des conventions sociales: il s'agit par exemple de Lebeau, ami de la famille Boniface, qui jette à la figure de Boniface père, ses fautes; il a non seulement couché avec la femme de celui-ci, mais aussi avec sa fille aînée Christine, dont on fête la noce, et sa fille cadette, Mariette, âgée de 14 ans. Au lieu de se taire ou d'éprouver, à défaut de honte, du moins un certain embarras, il se vante de ses exploits sexuels. C'est encore Bouc, le docteur de la famille, âgé de 80 ans, qui court après tous les jupons, avec succès. Dans Auto-da-fé, par contre, nous assistons en la personne de Kien à un refus, une négation totale du corps et de ses fonctions. Il y a plusieurs raisons à cela : tout d'abord Kien revit le mystère christique (c'est la «révélation » au mont de piété où il s'identifie au sauveur p.292, voir infra la symbolique de son nom): il abandonne donc son corps, car l'esprit domine, mais il donne également de son vivant son corps à la science puisqu'il a déjà donné son cerveau à un laboratoire pour en prouver les dimensions extraordinaires. Ce refus du corporel se manifeste également par son anorexie caractérisée, son état de délabrement physique (voir les descriptions qui sont faites de lui p. 11, 17, 26, 38, 528-529), le désintérêt pour son apparence. Il refuse également les fonctions corporelles d'ingestion ("Kien s'interdisait la faim et tous autres désagréments corporels ", p. 197) et de déjection (il a inventé une table de toilette à roulettes afin d'être débarrassé au plus vite de tout souvenir de son corps). Il sera intéressant de remarquer que Kien considère son corps comme un réceptacle clos : il refuse toute ouverture, toute brèche dans cette enveloppe fragile. Il refuse de recevoir ce qui est étranger, ce qui est autre de même qu'il dénie au monde une part de lui-même qu'il ressent comme une perte vitale. Pour lui, le fantasme de l'entropie est son seul espoir. C'est le fantasme de l'autarcie physique qui, il s'en apercevra à ses dépens avec le concierge Pfaff, le met dans une position bien vulnérable sur un terrain autre qu'intellectuel.

15 Cette dénégation du corporel le mène à une peur face aux réactions de son corps : ainsi lorsque Thérèse se déshabille devant lui pendant leur «lune de miel», il a trois types de réactions. Tout d'abord, il appelle son frère à l'aide - soit-disant parce que c'est un Don Juan, mais en fait parce que le rappel de la parenté lui permet de retomber en enfance d'où toute sexualité serait bannie. Ensuite, devant son incapacité à passer à l'acte et à consommer son mariage, il se réfugie dans les toilettes, où après avoir baissé son pantalon, il se met à sangloter toute la nuit : on pourrait parler ici d'une régression 
vers un stade antérieur à toute sexualité adulte. Enfin, il s'agit aussi d'une négation pure et simple de ses réactions physiques «involontaires " : il fixe son attention sur la jupe bleue de Thérèse et s'offusque de sa "raideur ", en affirmant qu' " une raideur exagérée est ridicule» (p. 64). Comment ne pas voir ici une autre raideur qui l'effraie.

La dernière manifestation de ce refus du corps se trouve également dans le nom de Kien, mais aussi dans son fantasme de la pétrification qui lui permettrait d'échapper aux agissements de Thérèse. Dieter Dissinger donne une interprétation des connotations de ce nom et de ce prénom ${ }^{6}$ pour ce qui nous intéresse ici, le fait que Peter (Pierre) renvoie à la succession du Christ et au martyre et à la raideur psychologique et physique du personnage qui se transforme en statue de pierre afin d'échapper aux coups que lui assène Thérèse ("Alors Kien se pétrifia» p. 195). Il y a de nouveau passage de l'animé à l'inanimé, de l'animal au minéral.

Ce refus du corps dégénère en violence physique et en cruauté, par moments difficilement supportables. C'est par exemple l'épisode qui clôt la seconde partie d'Autoda-fé où le Knopflians, qui joue l'aveugle dans la rue pour gagner sa vie - les infirmes sont d'ailleurs pléthore dans le roman -, empoigne le nain Fischerle caché sous le lit et s'emparant d'un couteau lui coupe avec jouissance sa bosse avant de se tourner vers la femme du nain. Et le narrateur de conclure : « Il pousse le cadavre sous le lit. Ensuite, il se jette sur la femme. «Personne n'a rien entendu » dit-il et il rit. Il est las, mais la femme est grasse. Il fait l'amour toute la nuit. » (p. 438) Ou bien c'est Pfaff, le concierge, qui a honte d'être roux et qui bat femme et enfant à mort afin d'échapper à ce complexe qui le hante, ou encore Kien qui se mutile après avoir tué les canaris du concierge (p. 528-529). Dans Noce, ce sont les enfants Boniface qui voient dans leur père une barrière à leurs débordements sexuels et qui l'assomment tous ensemble avec un fauteuil.

18 Ce refus du corps physique est aussi un refus du corps social, un refus de s'intégrer dans la société, de faire partie d'un ensemble qui dépasse les frontières de l'individu. Dans Noce cela se traduit par le fait que la maison se transforme en prison dont les issues sont fermées: Boniface barre la porte de l'appartement afin d'empêcher quiconque de quitter les lieux, ce qui se passe à l'extérieur ne peut être que menaçant et mortel, alors que ce qui se passe à l'intérieur l'est encore plus, mais chacun refuse de le voir, même s'il est pris de panique à cette idée. En ce qui concerne Auto-da-fé, c'est évidemment la métaphore filée de l'aveuglement («Blendung ») qui traduit au mieux cette négation. Pour Kien, il s'agit à la fois de l'enfermement dans la bibliothèque, mais aussi de la structure de son délire paranoïaque ${ }^{7}$ : le monde extérieur et la société dans son ensemble ne sont perçus que comme des menaces dirigées directement contre lui.

Nous voici maintenant arrivés à la fin de notre parcours, rapide certes, mais révélateur de la faillite de la conception du Moi issue des Lumières. En effet, ces deux œuvres de Canetti sont la traduction d'une prise de conscience de la crise de l'identité du sujet. Celui-ci ne peut plus prétendre à une vision totale et maîtrisée de la réalité - réelle ou fictive -, car pour Canetti, le mythe de la totalité humaine est un mythe périmé8. Comment ne pas voir ici une influence des théories de Ernst Mach sur le « unrettbares Ich » par exemple, mais aussi le refus de Canetti de se soumettre aux théories alors à la mode, tel le freudisme qu'il récuse tant chez Thomas Mann que chez Hermann Broch ou l'approche post-moderne éclatée telle qu'elle apparaît chez Döblin? Roger Gentis ${ }^{9}$ écrit : «Traqué dans son intériorité même, piégé dans le factice qui se donne à voir, le sujet s'épuise en une lutte quotidienne, dérisoire, pour tenter de se reconnaitre on ne 
sait où. Un des plus grands désastres écologiques qui se produise sous nos yeux est peut-être cette dévastation aveugle de l'espace subjectif. » Musil, lui, parle de « l'ombre grammaticale » du $\mathrm{Moi}^{10}$. Pour Canetti, cette mise en cause du Moi ne signifie pas une destruction ou plus exactement une disparition du Moi, mais bien plus la possibilité de lui rendre son élasticité, de parer à toute pétrification. En effet la foi que les Lumières ont mises dans le Moi, sa liberté, son affirmation intempestive au détriment de l'autre, ne pouvait conduire, conjuguée à une scientifisation de plus en plus poussée du monde, qu'à un repli frileux sur le Moi, dans cette forteresse assiégée où l'individu peut se croire vainement en sécurité. Mais ce que Canetti donne à voir dans les deux œuvres de notre analyse et qu'il développera plus tard dans Masse et puissance, c'est que cette «hibernation » du Moi le rend en fait plus vulnérable aux phénomènes de masse qui caractérisent le $\mathrm{xx}^{\mathrm{e}}$ siècle. Et Canetti ne se cachera jamais d'avoir perçu cette apparition des masses comme le seul phénomène digne d'intérêt de notre siècle et de notre monde occidental. C'est donc bien un procès qu'il intente à la vision du Moi proposée par les Lumières en en mettant au jour les dangers inhérents dont les conséquences les plus néfastes se sont fait sentir un siècle et demi plus tard. En1935, Canetti ne propose pas encore de remède ou de solution, il s'agit bien plus d'un bilan sobre et sarcastique d'un certain "malaise dans la civilisation ». Il nous montre également à quel point des entités si contraires, poussées à l'extrême, se ressemblent, comment la frontière entre les deux disparaît et comment imperceptiblement on bascule de l'un dans l'autre, comme il le montrera et le développera dans Masse et puissance à propos de l'individu et de lamasse.

\section{NOTES}

1. Elias Canetti, Hochzeit, Komödie der Eitelkeit, Die Befristeten, Frankfurt am Main, Fischer Taschenbuchverlag, 1987 (1964) ; Canetti, Elias, Théâtre : Noce, La comédie des vanités. Les sursitaires, traduit de l'allemand par FrançoisRey et Heinz Schwarzinger, Paris, Albin Michel, 1986, coll. Les Grandes Traductions.

2. Canetti, Elias, Die Blendung, Frankfurt am Main, Fischer Taschenbuchverlag, 1988 (1949); Canetti, Elias, Auto-da-fé, traduit de l'allemand par Paule Arhex, Paris Gallimard, 1972 (1968), coll. L'imaginaire.

3. On se rappellera l'importance de l'incendie du Palais de justice de Vienne le 25 juin 1927 par exemple, voir Le flambeau dans Voreille, Histoire d'une vie 1921-1931, traduit par Michel-François Démet, Albin Michel, Paris,1982, Les Grandes Traductions/Documents.

4. Pöder, Elfriede, "Spurensicherung. Otto Weiniger in der Blendung. », in : Aspetsberger F. \& Stieg G. (Hrsg.), Elias Canetti. Blendung als Lebensform, Königstein/Ts, Athennäum, 1985, pp. 57-62 ; Foell, Christie A., « Whores, Mothers, and others : reception of Otto Weiniger's Sex and Character in Elias Canetti's Autodafé », in : Horowitz, Nancy, A. \& Hyams, Barbara (Ed.), Jews and Gender-Responses to Otto Weiniger, Philadelphia Temple Univ., 1995, pp. 245-302 ; Stieg, Gerald, "Canetti und Kraus", Modern Austrian Literature 16, n $3 / 4$ "Special Canetti Issue", Binghampton (Calif.), p. 205.

5. J.-J. Lecercle, The Violence of Language, Routledge, London New-York,1990. 
6. Dissinger, Dieter, « Der Roman Die Blendung », in : Arnold, HeinzLudwig (Hrsg.), Elias Canetti, München, Edition Text+Kritik, 1982, p. 33-42.

7. Voir Canetti, Elias, Masse et puissance, Le cas Schreber, p. 461-494.

8. Meyer, Christine, Canetti, lecteur de Cervantès, Gogol, Stendhal: formes de l'intertextualité dans l'œuvre romanesque et autobiographique d'Elias Canetti, Thèse de doctorat, 1997, p. 202-213.

9. Gentis, Roger, La folie Canetti, Paris, Maurice Nadeau, 1992, p. 159.

10. Cometti, J.-R., Robert Musil ou l'alternative romanesque, Paris, PUF, 1985, coll. Perspectives critiques, p. 25.

\section{RÉSUMÉS}

Dans deux de ses œuvres de jeunesse, Noce (1932) et Auto-da-fé (1935), Elias Canetti nous montre à quel point les hommes malgré leur prétention à dominer la langue s'en retrouvent en fait les victimes. Ainsi le perroquet dans Noce qui répète sans cesse le mot «maison » ne comprend pas ce qu'il dit et ne sait pas qu'il s'agit du mot crucial qui fait s'agiter tous les personnages autour de lui. Dans Auto-da-fé les personnages tentent de maîtriser les autres en leur donnant un nouveau nom, sans s'apercevoir que dans cette entreprise, c'est la langue qui détermine leurs désirs. La qualité performative de la langue leur permet certes de faire exister leurs désirs, mais ce, dans une réalité virtuelle, dont ils deviennent tôt ou tard les victimes. Le corps et ses désirs sont soit réprimés, soit exaltés ce qui débouche dans les deux cas sur une violence physique qui se tourne aussi bien contre les autres que contre soi-même. Ainsi Elias Canetti nous fait assister à la faillite du Moi que les Lumières nous ont livré : son désir de maitrise absolue de soi et des autres le rend en fait vulnérable à ses pires ennemis tel le phénomène de la masse, ce qu'Elias Canetti développera dans Masse et puissance bien des années plus tard.

In zwei seiner frühen Werke, Hochzeit (1932) und Die Blendung (1935), zeigt Elias Canetti, wie die Menschen, die sich zu der Behauptung erdreisten, sie seien Herr der Sprache, eigentlich zu deren Opfer werden: Die Papagei in Hochzeit, die ständig das Wort «Haus » wiederholt, ohne die Bedeutung des Wortes und seine Tragweite zu verstehen, versinnbildlicht diesen Stand der Dinge. In der Blendung finden wir die verzweifelten Versuche der Figuren, durch die Sprache die Wirklichkeit zu meistern. Gestalten werden von anderen umbenannt, um sie zu beherrschen, ohne sich dessen bewußt zu sein, wie sehr sie dabei Spielball der Sprache selbst werden. Das beste Beispiel dafür liefert Therese. Parallel dazu wird der Körper mit seinem Begehren entweder verdrängt oder exaltiert, was in bei den Werken in eine Entladung von physischer Gewalt gegen die anderen und sich selbst mündet. Beide Werke veranschaulichen das Scheitern der durch die Aufklärung geprägten Auffassung des Ich. Die übertrieben angestrebte Selbständigkeit des Ich zu Kosten des Anderen macht es für Phänomene wie das der Masse anfällig, die letzten Endes seinen Untergang herbeiführen, wie Elias Canetti es viele Jahre später in Masse und Macht entwickelt hat.

\section{AUTEUR}

\section{ÉRIC LEROY DU CARDONNOY}

Université de Caen 